\title{
Association between DNA methylation and multidrug resistance in human glioma SHG-44 cells
}

\author{
JIN CHEN, ZHONG-YE XU and FENG WANG
}

Department of Neurosurgery, The Second Affiliated Hospital of Chongqing Medical University, Chongqing 400010, P.R. China

Received November 11, 2013; Accepted June 11, 2014

DOI: $10.3892 / \mathrm{mmr} .2014 .2690$

\begin{abstract}
The aim of the present study was to evaluate the association between DNA methylation and multidrug resistance (MDR) in glioma and identify novel effectors responsible for MDR in human gliomas. An MDR glioma cell line, SGH-44/ADM, was developed using adriamycin (ADM) impulse treatment. Cryopreservation, recovery and withdrawal were performed to evaluate the stability of SGH-44/ADM cells. The adherence rate and cellular morphology were observed by microscopy, and the cell growth curve and doubling time were determined. DNA methylation was analyzed using a methylated DNA immunoprecipitation microarray chip (MeDIP-Chip). The cell cycle, Rh123 ingestion and exudation, and SGH-44/ADM apoptosis were analyzed by flow cytometry. SGH-44/ADM cells showed little difference as compared with parental cells, except that SGH-44/ADM cells were bigger in size with a wizened nucleus. Compared to SGH-44 cells, a larger proportion of SGH-44/ADM cells remained in $\mathrm{G} 1$ and $\mathrm{S}$ phase, as measured by flow cytometry. The MDR of SGH-44/ADM was associated with the upregulation of multi-drug resistance 1 , prostaglandin-endoperoxide synthase 2 (COX-2); protein kinase $\mathrm{C} \alpha(\mathrm{PKC} \alpha)$; however, the expression of these genes was not associated with DNA methylation. In the MeDIP-Chip analysis, 74 functions were markedly enhanced, and seven significant pathways were observed. Genes including SNAP47, ARRB2, PARD $6 B$, $T G F B 1, V P S 4 B$ and $C B L B$ were identified by gene ontology analysis. The predominant molecular mechanism of MDR in SGH-44/ADM cells was identified as exocytosis and efflux. The expression of COX-2, PKC $\alpha$ and P-glycoprotein (Pgp) was not found to be associated with DNA methylation. Genes including SNAP47, VAMP4 and VAMP3 may serve as the downstream effectors of Pgp, COX-2 or PKC $\alpha$; however, further experiments are required to verify these observations.
\end{abstract}

Correspondence to: Dr Zhong-Ye Xu, Department of Neurosurgery, The Second Affiliated Hospital of Chongqing Medical University, 74 Linjiang Road, Chongqing 400010, P.R. China

E-mail: zhongyexu22@hotmail.com

Key words: DNA methylation, multidrug resistance, gliomas, SGH-44, adriamycin

\section{Introduction}

A glioma is a tumor that originates in the brain or spine, arising from glial cells. The most common site of gliomas is in the brain (1). Gliomas attribute for $\sim 30 \%$ of all brain and central nervous system tumors, and $\sim 80 \%$ of all malignant brain tumors. The anthracyclines, such as adriamycin (ADM), are among the most effective anticancer treatments, and are effective against more types of cancer than any other class of chemotherapeutic agents (2-4). However, the effect of chemotherapy on the survival rate in patients with malignant glioma is low, giving an improvement in survival rate of $6 \%$ after one year and 5\% after two years (5). This poor response to therapy may be due to multi-drug resistance (MDR) (6).

Mechanisms to explain drug resistance have been reported to include the blood-brain barrier (BBB), efflux pumps, DNA repair and cancer stem-like cells (7-9). P-glycoprotein (Pgp), the MDRl gene product in humans, is a drug efflux pump which has a significant role in modulating MDR in a wide variety of human cancers $(10,11)$. Pgp is highly expressed in the BBB, with weak expression in tumor cells (12). MDR-associated protein 1 (MRP1), expressed in glioma cell lines, has been suggested to be involved in MDR both in vivo and in vitro $(13,14)$. Low density lipoprotein receptor-related protein (LRP) is a member of the low density lipoprotein (LDL) receptor (15). A previous study showed that the association of statins, plus an LDL receptor-targeted liposomal drug, may increase in vitro drug delivery across the BBB (16). It has been previously observed that the expression levels of prostaglandin-endoperoxide synthase 2 (COX-2), protein kinase C $(\mathrm{PKC})$ and Activator Protein 1 were significantly upregulated in drug resistant cell lines that overexpress MDR1/Pgp170, suggesting that there is a potential link between COX-2, PKC and MDR1/Pgp170 (17).Glutathione S transferase $\pi$ (GST $\pi$ ) is another protein that catalyzes conjugation reactions of glutathione (GSH), linking to cytotoxic drugs and leading to their inactivation (18). In addition, the Fanconi anemia (FA) pathway is involved in glioma drug resistance (19).

The pattern of methylation is an important line of research in MDR (20-22). Recent research has demonstrated that acquired gemcitabine resistance is associated with DNA promoter methylation-independent human equilibrative nucleoside transporter 1 and deoxycytidine kinase gene downregulation and hyper-expression of G9A methyltransferase (21). The death-associated protein kinase (DAPK) is 
hypermethylated in drug-resistant derivatives generated from both non-small cell lung cancer (NSCLC) and head and neck squamous cell carcinoma (HNSCC) cell lines (22). Expression of O6-methylguanine-DNA methyltransferase is associated with resistance to radiotherapy in gliomas (23). However, the association between methylation and MDR in glioma has yet to be evaluated.

In the present study, a stable MDR glioma cell line, SGH-44/ADM, was generated and the association between DNA methylation and MDR was analyzed using a methylation DNA immunoprecipitation microarray chip (MeDIP-Chip). The findings from this study may provide novel insight for the treatment of gliomas.

\section{Materials and methods}

Cell culture and construction of an ADM resistant SGH-44/ADM cell line. The human glioma cell line SHG-44 (Medicine College of Suzhou University, Suzhou, China) was routinely cultured in RPMI 1640 medium (Gibco-BRL, Invitrogen Life Technologies, Carlsbad, CA, USA) supplemented with 5 or $10 \%$ heat-inactivated fetal bovine serum (FBS; HyClone Laboratories, Inc., Logan, Utah, USA), $100 \mathrm{U} / \mathrm{ml}$ penicillin and $100 \mathrm{U} / \mathrm{ml}$ streptomycin (Qilu Pharmaceutical Co., Ltd., Jinan,China). An ADM-resistant cell line of SHG-44, named as SHG-44/ADM, was obtained by culturing the cells in the presence of gradually increasing doses of ADM (Shenzhen Main Luck Pharmaceuticals Inc., Shenzhen, China). In brief, SHG-44 cells were cultured in RPMI 1640 medium with $0.01 \mathrm{mg} / \mathrm{ml}$ ADM. The medium was removed and replaced after $48 \mathrm{~h}$, and the cells were cultured to resume normal growth. The cells were then transferred and treated with $0.1 \mathrm{mg} / \mathrm{ml}$ ADM. The above process was repeated to obtain ADM-resistant cells. After eight months, cells cultured in $0.1 \mu \mathrm{M}$ ADM were harvested as the ADM-resistant cell line.

Antitumor drug resistance of SHG-44 and SGH-44/ADM. The proliferation inhibition of simvastatin on glioma cell lines was determined using a WST-8 Cell Counting Kit-8 (CCK-8; Beyotime Institute of Biotechnology, Haimen, China). The cells were seeded in fresh RPMI 1640 medium containing $10 \%$ fetal calf serum $24 \mathrm{~h}$ prior to the experiments. Cells were seeded at an initial concentration of $1 \times 10^{5}$ cells $/ \mathrm{ml}$ in $100 \mu \mathrm{l}$ medium in 96-well culture plates with various concentrations of ADM, epinephrine (EPI; Harvest Pharmaceutical Co., Ltd., Shanghai, China), vincristine (VCR; Shenzhen Main Luck Pharmaceuticals Inc.), mitomycin C (MMC; Nanjing Duoyan Biochemistry Co., Ltd., Nanjing, China), arabinofuranosyl cytidine (Ara-C; Nanjing Duoyan Biochemistry Co., Ltd.,) and cisplatin (DDP; Qilu Pharmaceutical Co., Ltd.) for $72 \mathrm{~h}$. Then $20 \mu \mathrm{l}$ of CCK-8 staining solution was added to each well for $2 \mathrm{~h}$ at $37^{\circ} \mathrm{C}$ before the end of the incubation. The absorbance at $450 \mathrm{~nm}$ was measured using a microplate reader (Model 550; Bio-Rad Laboratories, Inc., Hercules, CA, USA). The half inhibitory concentration $\left(\mathrm{IC}_{50}\right)$ and resistance index $(\mathrm{RI})$ were calculated.

Effect of cryopreservation, recovery and withdrawal on the RI. SHG44/ADM cells were stored in liquid nitrogen, recovered after three months, and then the RI was measured. In addition, the RI of SHG44/ADM cells, which were cultured without ADM for one month, was also measured.

Analysis of adherence rate, cellular morphology, cell growth curve and doubling time. The cellular morphology was observed using an inverted microscope (IX-71, Olympus Corporation, Tokyo, Japan). Cells were seeded at an initial concentration of $1 \times 10^{5}$ cells $/ \mathrm{ml}$ in $24-w e l l$ culture plates. The pelagic cells were counted every two hours in an eight-hour period. The adherence rate (AR) was calculated as $\left(1-\mathrm{C}_{\mathrm{ad}} / \mathrm{C}_{\text {total }}\right) \times 100 \%$. Cells were seeded at an initial concentration of $5 \times 10^{5} \mathrm{cell} / \mathrm{s} / \mathrm{ml}$ in 24-well culture plates, and the viable cells were counted every day for one week. The cell growth curve was constructed, and the doubling time was calculated using the Patterson formula: $\mathrm{Td}=\mathrm{T} \times \lg 2 /(\operatorname{gN} 2-\lg \mathrm{N} 1)$. Where $\mathrm{Td}$ is doubling time; $\mathrm{T}$ is time interval; N2 is end point cell number; and N1 is initial cell number.

Double layer soft agar detection for colony formation. Cells were diluted to 125,250 and 500 cells/ml with culture medium, respectively. The diluted cells were then mixed 1:1 with $0.7 \%$ agar and poured on $1.2 \%$ concretionary agar. When the upper layer was curdled, the cells were cultured for seven days at $37^{\circ} \mathrm{C}$ with $5 \% \mathrm{CO}_{2}$. The colony with $>50$ cells was counted.

Flow cytometry for cell cycle analysis. The measurement of the cell cycle was performed by flow cytometry (BD FACSCalibur $^{\mathrm{TM}}$, BD Biosciences, Franklin Lakes, NJ, USA). After synchronization, the cells were cultured in fresh medium with $10 \%$ FBS to $90 \%$ confluency, harvested and suspended with $0.3 \mathrm{ml}$ phosphate buffered saline (PBS) containing 5\% FBS. A volume of $0.7 \mathrm{ml}$ ethanol was added for immobilization. The cells were stored at $-20^{\circ} \mathrm{C}$ for $24 \mathrm{~h}$ and washed with PBS. RNase A, $0.1 \mathrm{mg}$, was added for RNA degradation, then the cells were placed in the dark for 10 min after adding $0.2 \mu \mathrm{g}$ propidium iodide (PI). The percentages of cells in different phases were detected by flow cytometry.

Methylated DNA immunoprecipitation microarray chip (MeDIP-Chip) and analysis. The DNA of SHG-44 and SHG-44/ADM was extracted using a DNA extraction kit (Qiagen, Hilden, Germany). The methylated DNA was purified using EpiQuik Methylated DNA Immunoprecipitation Kit (Qianchen Biotechnology Company, Shanghai, China). The target DNA was amplified with primer A and digested with $U d p$ and ApeI restriction enzymes. The DNA was mixed with $12 \mu 1$ TdT buffer, $2 \mu 1$ TdT and $1 \mu 1$ DNA Labeling reagent. The mixture was used for gel shift analysis. The data analysis was performed as previously described (24).

Pathway analysis of MeDIP-Chip. Pathway analysis was performed according to the Kyoto Encyclopedia of Genes and Genomes (KEGG; www.genome.jp/kegg/), BioCarta (www. biocarta.com) and Reactome (www.reactome.org). In addition, Fisher's exact test and $\chi^{2}$ test was performed to select the significant pathways, and the threshold of significance was defined by P-value and false discovery rate (FDR). The enrichment $(\mathrm{Re})$ was calculated as $\operatorname{Re}=\left(\mathrm{n}_{\mathrm{f}} / \mathrm{n}\right) /\left(\mathrm{N}_{\mathrm{f}} / \mathrm{N}\right)$, where $\mathrm{n}_{\mathrm{f}}$ was the number of flagged genes within the particular 
category, $\mathrm{n}$ was the total number of genes within the same category, $\mathrm{N}_{\mathrm{f}}$ was the number of flagged genes in the entire microarray and $\mathrm{N}$ was the total number of genes in the microarray (25-27).

Gene ontology $(G O)$ analysis of MeDIP-Chip. GO analysis was applied to analyze the main functions of the differentially expressed genes according to the GO, which was the key functional classification of the National Center for Biotechnology Information (NCBI), which can organize genes into hierarchical categories and uncover the gene regulatory network on the basis of biological processes and molecular function $(28,29)$. Specifically, a two-tailed Fisher's exact test and $\chi^{2}$ test was used to classify the GO category, and the FDR (30) was calculated to correct the P-value. The smaller the FDR, the smaller the error in judging the P-value. The FDR was defined as $F D R=1-N_{k} / T$, where $N_{k}$ referred to the number of Fisher's test P-values $<\chi^{2}$ test P-values. The P-values were computed for the GOs of all the differential genes. Enrichment provided a measure of the significance of the function. As the enrichment increased, the corresponding function was more specific. This helped identify those GOs with a conclusive function description in the experiment. Within the significant category, the enrichment Re was given by: $\operatorname{Re}=\left(n_{f} / n\right) /\left(N_{f} / N\right)$ with the variables defined as stated above (31).

Expression analysis of MDR 1, MRP1, LRP1, PKC $\alpha$ and COX-2. Total RNA of the lymph node was isolated using TRIzol ${ }^{\mathrm{TM}}$ reagent (Invitrogen Life Technologies). The reverse transcription reaction was conducted using random primers and the SuperScript III first-strand synthesis system (Invitrogen Life Technologies). The quantitative polymerase chain reaction (qPCR) was conducted using the following conditions: $95^{\circ} \mathrm{C}$ for $30 \mathrm{sec}$ followed by 40 cycles of $94^{\circ} \mathrm{C}$ for $5 \mathrm{sec}$ and $60^{\circ} \mathrm{C}$ for $30 \mathrm{sec}$, and dissociation curve analysis of the amplification products was performed at the end of each PCR reaction to confirm that only one product was amplified and detected. Each sample was run in triplicate together with the internal control gene, GAPDH. Data analysis of the qPCR was performed with Rotor Gene 6000 Series Software (Corbett Life Sciences, Sydney, Australia). The specific primer sequences (Invitrogen, Shnaghai, China) are listed in Table I.

The SGH-44/ADM and SGH-44 cell lines were washed three times with PBS and cell lysates were prepared in lysis buffer. Protein concentration was estimated using the bicinchoninic acid assay (Pierce Biotechnology, Inc., Rockford, IL, USA) and $20 \mathrm{mg}$ of protein was loaded in each lane. The proteins were resolved by $10 \%$ SDS-PAGE. The gel was transferred to a nitrocellulose membrane and blocked with 5\% non-fat dry milk (NFDM) for one hour at room temperature. The blot was then incubated with primary antibodies for overnight at $4{ }^{\circ} \mathrm{C}$ in $2.5 \%$ NFDM. Blots were washed with Tris-buffered saline containing $0.1 \%$ Tween 20 three times and subsequently incubated with horseradish peroxidase-conjugated goat-anti-rabbit immunoglobulin $\mathrm{G}$ antibody (Pierce Biotechnology, Inc.). Enhanced Chemiluminescence substrate (Pierce Biotechnology, Inc.) was used to detect the signal. The blots were stripped and re-probed with mouse monoclonal $\beta$-actin antibody, which served as an internal control. All the
Table I. Specific primers for quantitative polymerase chain reaction.

\begin{tabular}{ll}
\hline Genes & \multicolumn{1}{c}{ Primer sequence } \\
\hline PRPS1 & F: 5'-ATCTTCTCCGGTCCTGCTATT-3' \\
& R: 5'-TGGTGACTACTACTGCCTCAAA-3' \\
ARRB2 & F: 5'-TCCATGCTCCGTCACACTG-3' \\
& R: 5'-ACAGAAGGCTCGAATCTCAAAG-3' \\
GBA & F: 5'-CATCCGCACCTACACCTATGC-3' \\
& R: 5'-TGAGCTTGGTATCTTCCTCTGG-3' \\
GAPDH & F: 5'-GGAGCGAGATCCCTCCAAAAT-3' \\
& R: 5'-GGCTGTTGTCATACTTCTCATGG-3'
\end{tabular}

F, Forward; R, Reverse; PRPS1, phosphoribosyl pyrophosphate synthetase 1 ; ARRB2, arrestin $\beta 2$; GBA, glucocerebrosidase.

Table II. Resistance of SGH-44 and SGH-44/ADM to different drugs.

\begin{tabular}{lcrr}
\hline & \multicolumn{2}{c}{$\mathrm{IC}_{50}(\mu \mathrm{g} / \mathrm{ml})$} & \\
\cline { 2 - 3 } Drugs & $\mathrm{SGH}-44$ & $\mathrm{SGH}-44 / \mathrm{ADM}$ & $\mathrm{RI}$ \\
\hline ADM & $1.67 \pm 0.05$ & $17.83 \pm 0.52$ & 10.7 \\
EPI & $0.87 \pm 0.06$ & $7.22 \pm 0.14$ & 8.3 \\
VCR & $2.95 \pm 0.09$ & $21.42 \pm 0.67$ & 7.2 \\
MMC & $8.74 \pm 0.33$ & $47.73 \pm 1.12$ & 5.4 \\
Ara-C & $8.38 \pm 0.42$ & $10.27 \pm 0.39$ & 1.2 \\
DDP & $0.78 \pm 0.07$ & $2.46 \pm 0.11$ & 3.1 \\
\hline
\end{tabular}

$\mathrm{IC}_{50}$, half inhibitory concentration; RI, resistance index; $\mathrm{ADM}$, adriamycin; EPI, epinephrine; VCR, vincristine; MMC, mitomycin C; Ara-C, arabinfuranosyl cytidine; DDP, cisplatin; SGH-44/ADM, SHG-44 cell line with ADM resistance.

antibodies were purchased from Cell Signaling Technology, Inc. (Danvers, MA, USA).

Rhodamine-123 (Rh123) ingestion and exuding of SGH-44/ADM. The SGH-44 and SGH-44/ADM cells were diluted to $1 \times 10^{6}$ cells $/ \mathrm{ml}$ with medium. Rh123 (Sigma-Aldrich) was added to the cells to a final concentration of $1 \mu \mathrm{g} / \mathrm{ml}$. The cells were incubated at $37^{\circ} \mathrm{C}$ for $1 \mathrm{~h}$ and washed three times with PBS. The relative fluorescence intensity of Rh123 accumulated in the cells was measured by flow cytometry at $4^{\circ} \mathrm{C}$. To detect the Rh123 exudation, the cells were diluted with RPMI 1640 medium and incubated for $2 \mathrm{~h}$. Then the cells were washed three times with PBS and the relative fluorescence activity of Rh123 was measured again.

Apoptosis of SGH-44 and SGH-44/ADM. SGH-44 and SGH-44/ADM cells were collected and diluted in PBS. Annexin V-fluorescein isothiocyanate (FITC; Nanjing KeyGen 
A

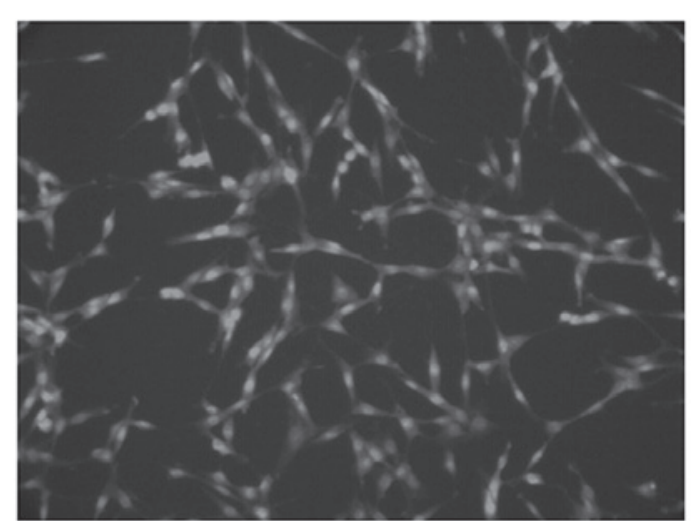

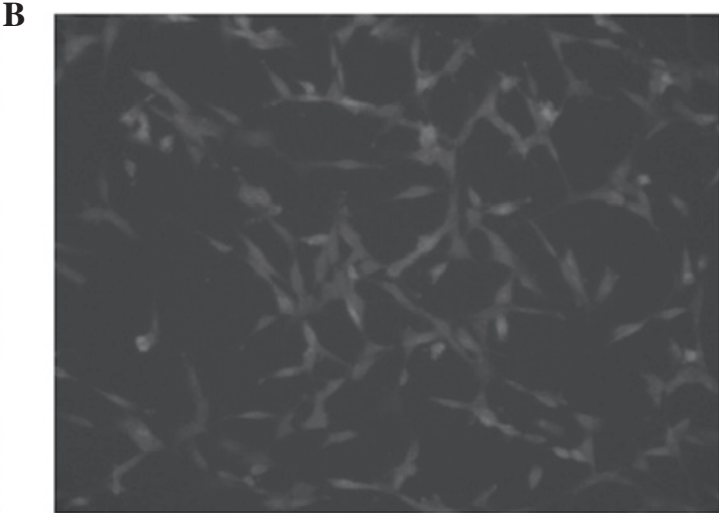

Figure 1. Cellular morphology of SHG-44 and SHG-44/ADM cells (magnification, x100). (A) SHG-44 and (B) SHG-44/ADM cells.SHG-44/ADM, human glioma cell line SHG-44 with adriamycin resistance.

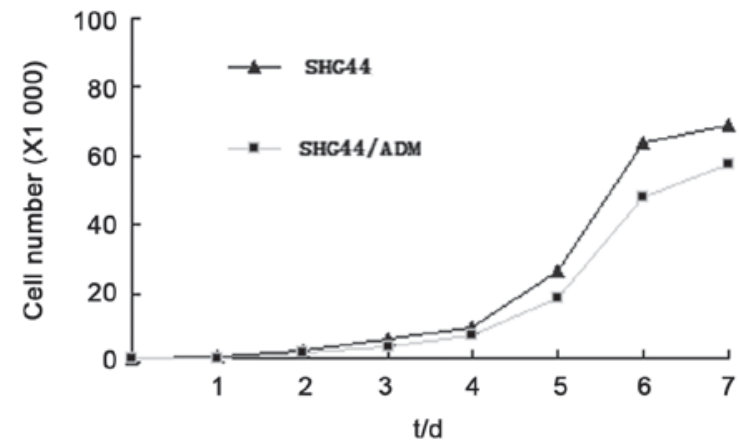

Figure 2. Cell growth curve of SHG-44 and SHG-44/ADM cells. t/d, generation time; SHG-44/ADM, human glioma cell line SHG-44 with ADM resistance; ADM, adriamycin.

Biotech. Co., Ltd., Nanjing, China), $195 \mu 1$, was added to $5 \times 10^{4}$ cells and the solution $(1 \mu \mathrm{g} / \mathrm{ml})$ was incubated in the dark for $10 \mathrm{~min}$ and centrifuged. Following this, $190 \mu \mathrm{l}$ Annexin V-FITC and $10 \mu \mathrm{l}$ propidium iodide (PI; Molecular Probes ${ }^{\circledR}$ Life Technologies, Carlsbad, CA, USA) was added. The solution was analyzed by flow cytometry. To evaluate the effect of MDR1 on the MDR of SGH-44/ADM, cyclosporin A (CsA; Sandoz, Holzkirchen, Germany), an MDR1 inhibitor, was added to the SGH-44/ADM cells. A fluorometric assay was performed as previously described (32).

\section{Results}

Antitumor drug sensitivities and stability of SGH-44/ADM. The $\mathrm{IC}_{50}$ to ADM of wild-type SGH-44 was $1.67 \mu \mathrm{g} / \mathrm{ml}$, while the $\mathrm{IC}_{50}$ to ADM of SGH-44/ADM was $17.83 \mu \mathrm{g} / \mathrm{ml}$. The RI of SGH-44/ADM was 10.7, and following freezing and recovery of the cells, the RI was 10.5. If the ADM was withdrawn, the RI of SGH-44/ADM was reduced to 9.3. The resistance of SGH-44/ADM to other antitumor drugs is shown in Table II.

Adherence rate, cellular morphology, cell growth curve and doubling time. The cell shape of SGH-44 and SGH-44/ADM cells is shown in Fig. 1. In the drug resistance formation process, the SGH-44 cells were shaped as elongated fibers, uniform in size, tightly packed, adherent to the plate and grown with a clear boundary. Upon administration of the drugs, the
A

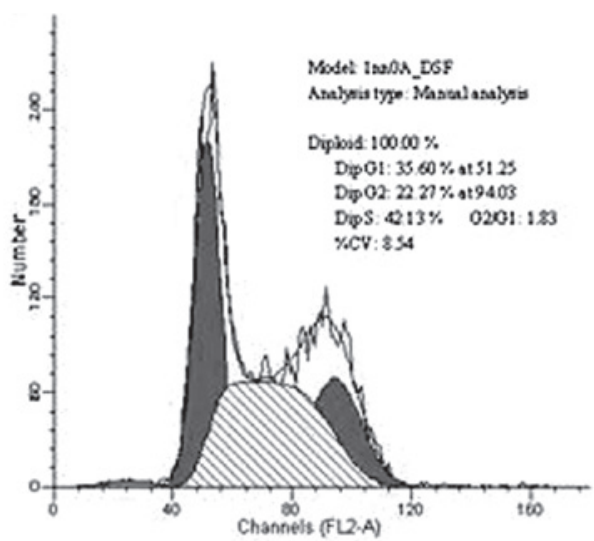

B

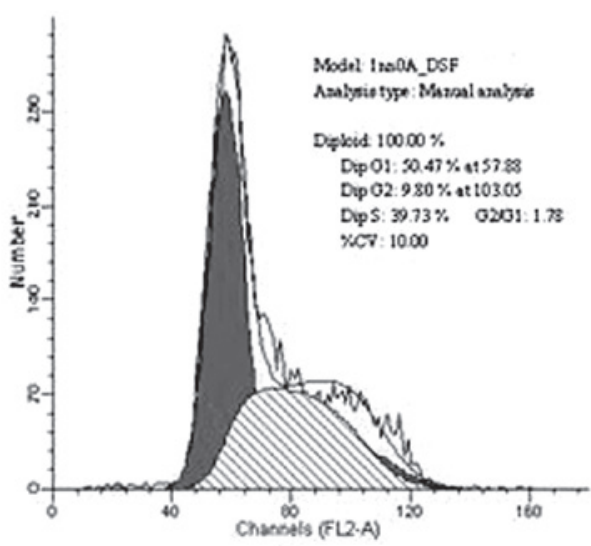

Figure 3. Cell cycle analysis of SGH-44 and SGH-44/ADM cells, measured by flow cytometry. (A) SHG-44 and (B) SHG44/ADM cells. SHG-44/ADM, human glioma cell line SHG-44 with adriamycin resistance.

surviving cells were not uniform in size, weakly adherent to the plate and shaped with a clear boundary. The most significant difference between the SGH-44 and SGH-44/ADM cells was that the SGH-44 cells had larger nuclei as compared with SGH-44/ADM cells. With extension of the incubation time and number of passages, this emergent abnormal state was reduced. Finally, SGH-44/ADM cells showed minimal differences as compared with parental cells, apart from SGH-44/ADM cells being bigger in size, and with a wizened nucleus.

The adherence rate was calculated in order to evaluate the viability of SGH-44/ADM cells. The adherence rate of SGH-44 


\section{Diffgene Sig GO}

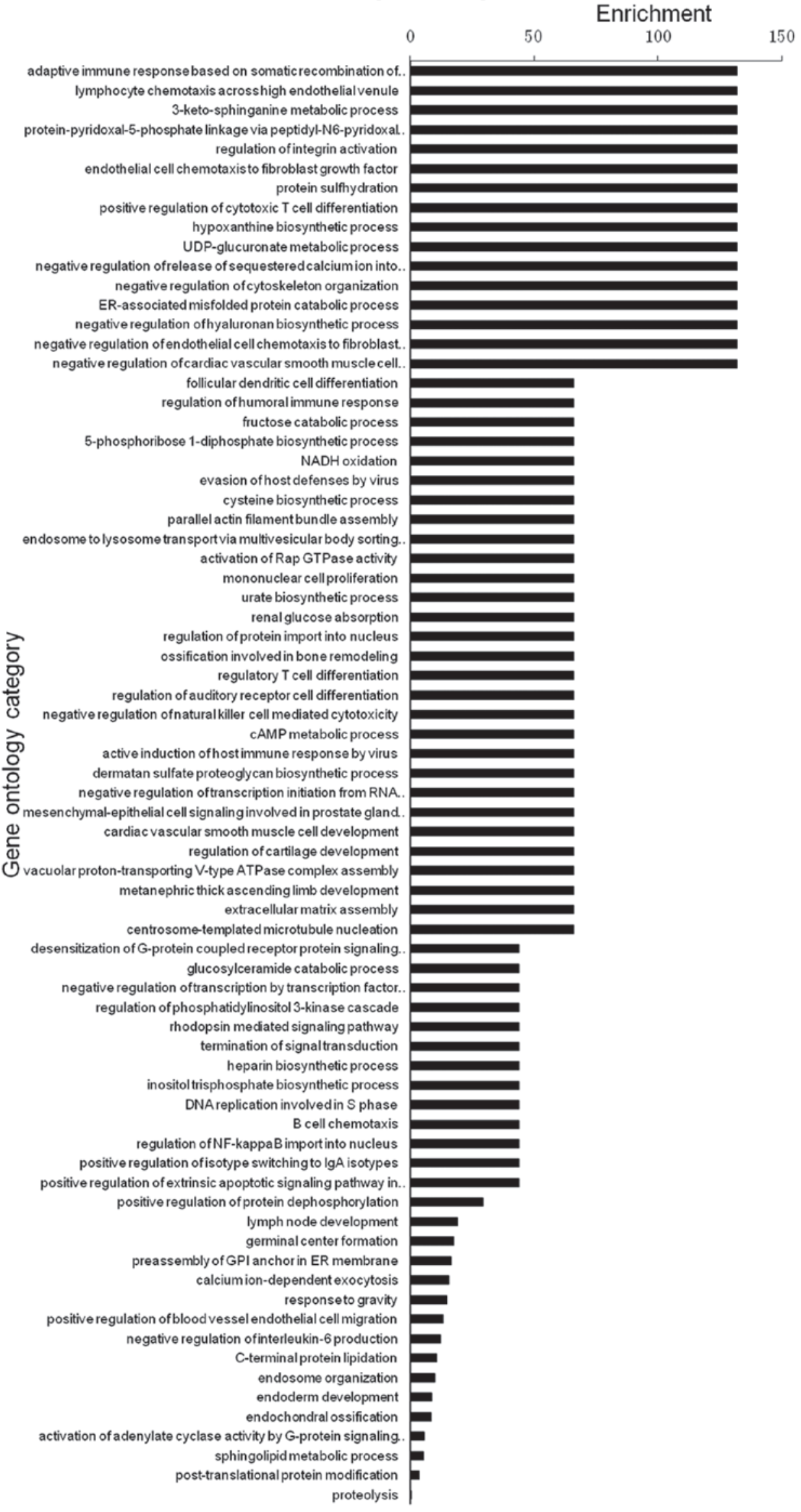

Figure 4. Functional enrichment of the differentially expressed genes, identified between SGH-44/ADM and SGH-44 cells (P<0.05). Sig GO, significant gene ontology; SHG-44/ADM, human glioma cell line SHG-44 with adriamycin resistance. 
cells at $0,2,4,6$ and $8 \mathrm{~h}$ was $0,68.5,86.6,93.5$ and $96.8 \%$, respectively. The adherence rate of SGH-44/ADM cells at 0 , $2,4,6$ and 8 h was $0,62.3,81.5,91.6$ and $94.7 \%$, respectively. The growth curve is shown in Fig. 2. SGH-44/ADM cells showed a slower growth rate as compared with parental cells. The doubling time of SGH-44/ADM was $32.22 \mathrm{~h}$ as compared with $26.67 \mathrm{~h}$ for SGH-44 cells.

Improvement of the rate of colony formation in $S G H-44 / A D M$ cells. The colony formation rate was calculated as the percentage of cells which could form small groups when dispersed into single cells. Double-layer soft agar experiments showed that the colony formation rate of SGH- 44 cells was $20 \%$, whereas the colony formation rate of SGH-44/ADM cells was significantly improved to $52 \%(\mathrm{P}<0.01)$.

Analysis of the cell cycle by flow cytometry. Flow cytometry was used to evaluate the percentage of SGH-44 and SGH-44/ADM cells in different phases of the cell cycle. The percentage of cells in G1 phase was increased from 35.6 to $50.4 \%$ when SGH- 44 acquired ADM resistance. The percentage of cells in S phase was 39.7\% in SGH-44/ADM cells (Fig. 3).

GO analysis by MeDIP-Chip. A functional enrichment analysis was performed using the Web-based Gene Set Analysis Toolkit $(33,34)$ and 74 significantly enhanced functions were identified (Fig. 4; FDR < 0.05). Immune-associated reactions, including the adaptive immune response based on somatic recombination of immune receptors built from immunoglobulin superfamily domains, lymphocyte chemotaxis across the high endothelial venule and positive regulation of cytotoxic T-cell differentiation were significantly enriched (enrichment $=131 ; \mathrm{P}<0.05)$.

Metabolic processes, including the 3-keto-sphinganine metabolic, hypoxanthine biosynthetic, UDP-glucuronate metabolic, endoplasmic reticulum-associated misfolded protein catabolic and negative regulation of hyaluronan biosynthetic processes were enriched (enrichment $=131 ; \mathrm{P}<0.05)$.

Pathway enrichment analysis by MeDIP-Chip. Pathway enrichment analysis of all genes involved in MeDIP-Chip was performed using the KEGG automatic annotation server. A total of seven significant pathways with $\mathrm{P}<0.05$ were enriched (Fig. 5). The most significant pathway was synaptosomal-associated protein (SNAP) receptor (SNARE) interaction in vesicular transport (path_id=4130), where $\mathrm{P}=0.005$. Three genes were involved in this pathway, including SNAP47, vesicle associated membrane protein $(V A M P) 4$ and VAMP3. The other significantly enriched pathways included sphingolipid metabolism, endocytosis, glycosylphosphatidylinositol (GPI)-anchor biosynthesis, collecting duct acid secretion, pentose phosphate pathway and glycine, serine and threonine metabolism. Genes associated with endocytosis included charged multivesicular body protein $1 \mathrm{~b}(C H M P 1 B)$, arrestin $\beta 2$ (ARRB2), par-6 family cell polarity regulator $\beta$ (PARD6B), transforming growth factor $\beta 1$ (TGFB1), vacuolar protein sorting 4 homolog $\mathrm{B}$ $(V P S 4 B)$ and cbl proto-oncogene, E3 ubiquitin protein ligase $\mathrm{B}(C B L B)$.

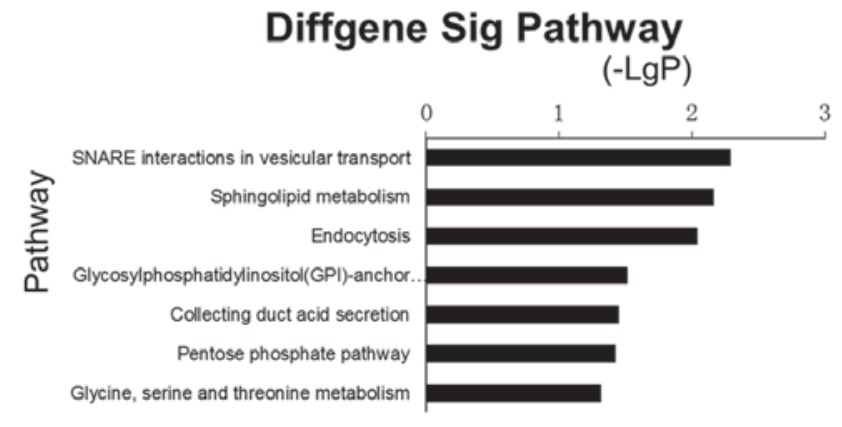

Figure 5. Pathway enrichment in MeDIP-Chip.

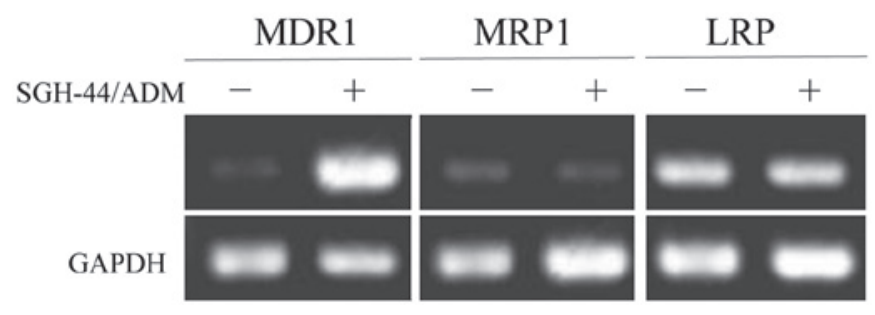

Figure 6. MDR1, MRP1 and LRP mRNA expression in SHG-44/ADM cells. MDR1, multidrug resistance 1; MRP1, MDR-associated protein 1; LRP, low density lipoprotein receptor-related protein 1 . SHG-44/ADM, human glioma cell line SHG-44 with adriamycin resistance.

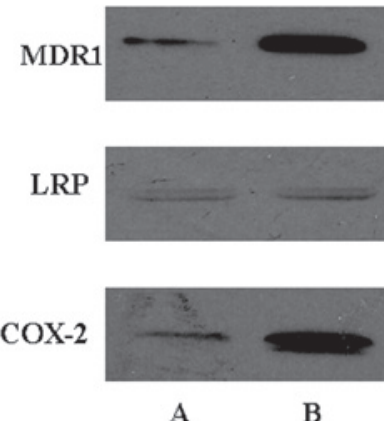

MRP1

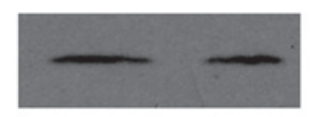

$\beta-\operatorname{actin}$

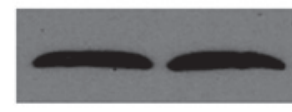

A

B

PKC $\alpha$

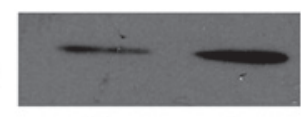

A

B

Figure 7. Protein expression of MDR1, MRP1, LRP, COX-2 and PKCa. (A) SHG-44 and (B) SHG44/ADM cells. MDR1, multidrug resistance 1; MRP1, MDR-associated protein 1; LRP, low density lipoprotein receptor-related protein 1; COX-2, prostaglandin-endoperoxide synthase 2; $\mathrm{PKC} \alpha$, protein kinase $\mathrm{C} \alpha$.

mRNA and protein expression levels of MDRI, MRPI and $L R P$. The expression of $M D R 1, M R P I$ and $L R P$ at the mRNA levels are shown in Fig. 6. The expression of MDRI at the mRNA level were significantly increased in SGH-44/ADM cells as compared with SGH-44 cells. The expression of MRP1 and LRP remained unchanged in the two cell lines at the mRNA and protein level. The expression of MDR1 at the protein level was increased in SGH-44/ADM cells as compared with SGH-44 cells, which was consistent with the mRNA expression levels. In addition, the expression of COX-2 and PKC $\alpha$ was increased in SGH-44/ADM cells. The protein expression levels are shown in Fig. 7.

Rh123 ingestion and exudation in SGH-44/ADM cells. An Rh123 ingestion and exudation experiment was performed by flow cytometry (Fig. 8). The amount of Rh123 intake 
A

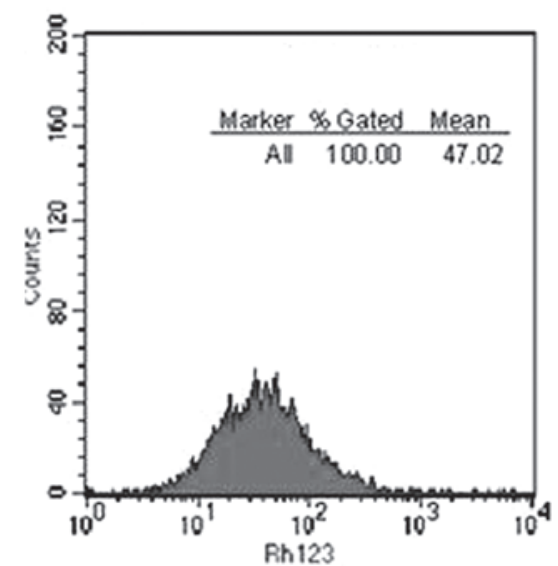

C

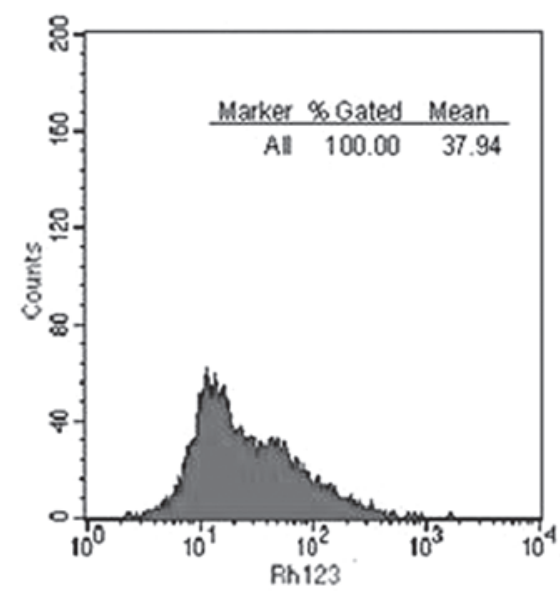

B

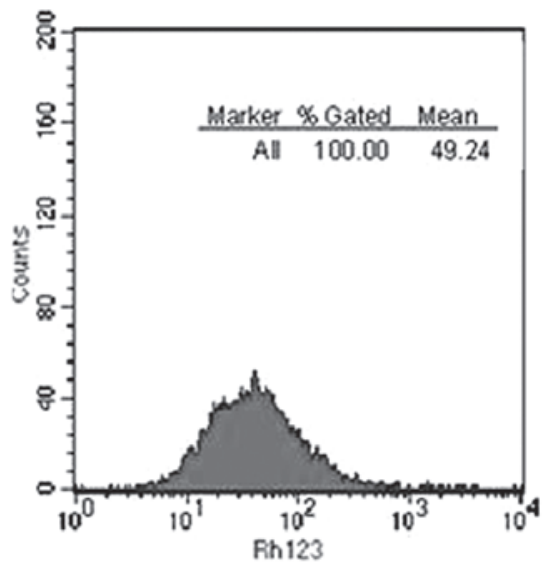

D

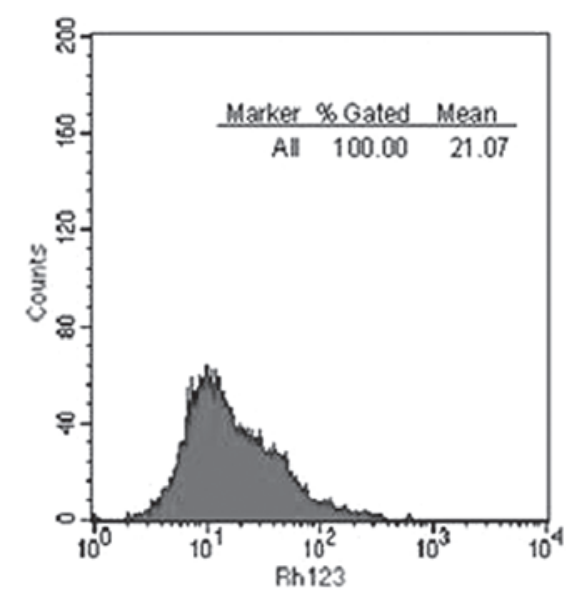

Figure 8. Rh123 accumulation in SGH-44 and SGH-44/ADM cells. (A) SHG-44 and (B) SHG-44/ADM cells treated with $1 \mu \mathrm{g} / \mathrm{ml}$ Rh123 for 1 h. (C) SHG-44 and (D) SHG-44/ADM cells treated with $1 \mu \mathrm{g} / \mathrm{ml} \mathrm{Rh} 123$ for $1 \mathrm{~h}$ and then cultured without Rh123 for $2 \mathrm{~h} . R \mathrm{~h} 123$, rhodamine-123; SHG-44/ADM, human glioma cell line SHG-44 with adriamycin resistance.

A

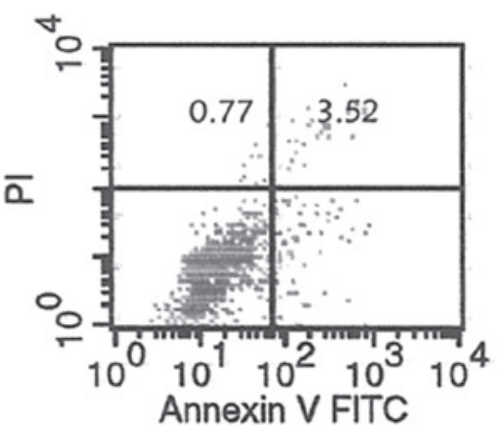

D

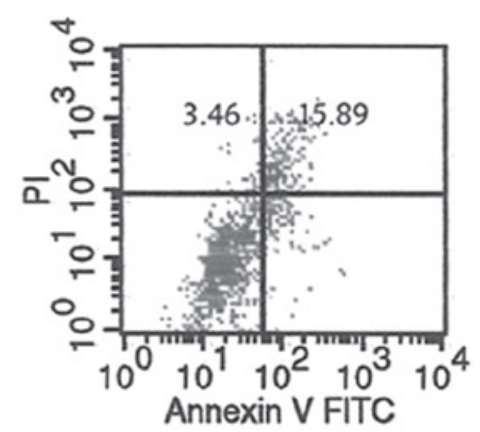

B

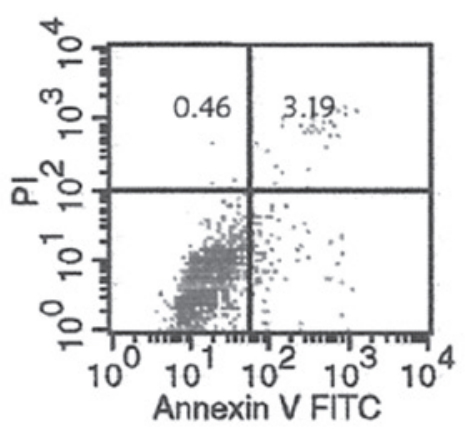

$\mathbf{E}$

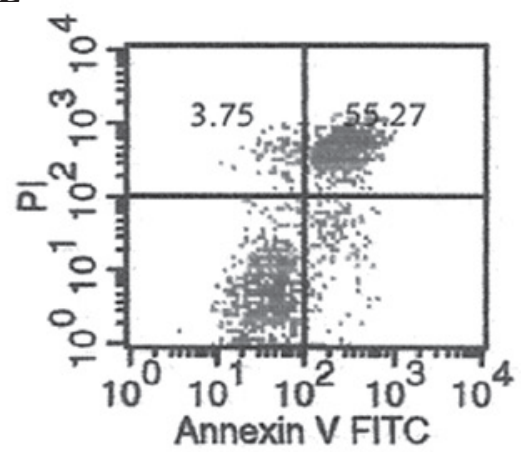

C

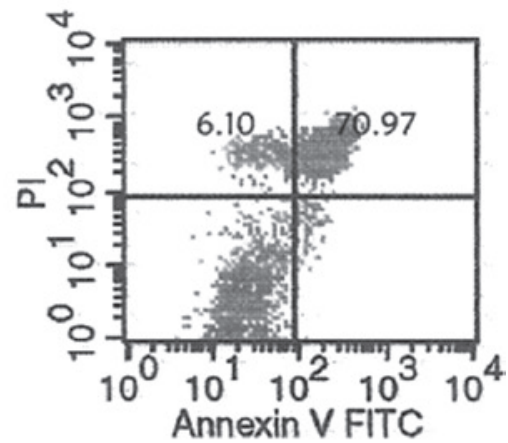

Figure 9. Cell apoptosis rate determined by flow cytometry. (A) SHG-44 and (B) SHG44/ADM cells without any treatment. (C) SHG-44 and (D) SHG44/ADM cells treated with $10 \mu \mathrm{g} / \mathrm{m} 1 \mathrm{ADM}$ for $48 \mathrm{~h}$. (E) SHG44/ADM cells treated with $10 \mu \mathrm{g} / \mathrm{m} 1 \mathrm{ADM}$ and $100 \mu \mathrm{M}$ cyclosporin A for $48 \mathrm{~h}$. ADM, adriamycin; FITC, fluorescein isothiocyanate; PI, propidium iodide; SHG-44/ADM, human glioma cell line SHG-44 with adriamycin resistance. 


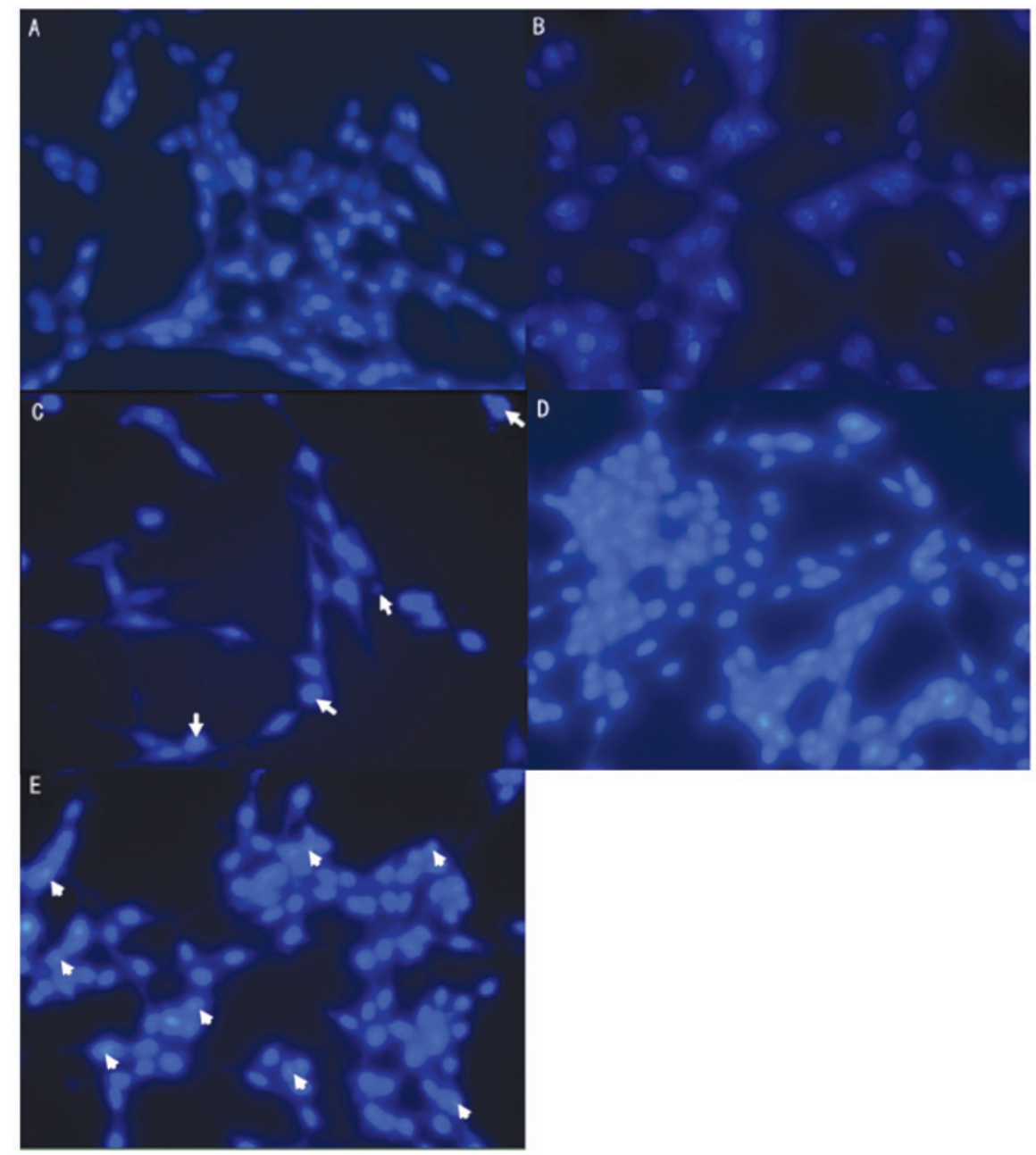

Figure 10. Apoptosis of SGH-44 and SGH-44/ADM determined by Hoechst 33258 staining and fluorescence microscopy (magnification, X100). (A) SHG-44 and (B) SHG-44/ADM cells without any treatment. (C) SHG-44 and (D) SHG-44/ADM cells treated with $10 \mu \mathrm{g} / \mathrm{ml}$ ADM for 48 h. (E) SHG-44/ADM cells treated with $10 \mu \mathrm{g} / \mathrm{ml} \mathrm{ADM}$ and $100 \mu \mathrm{M}$ cyclosporin A for $48 \mathrm{~h}$. Cells undergoing apoptosis are indicated by arrows. ADM, adriamycin; SHG-44/ADM, human glioma cell line SHG-44 with adriamycin resistance.

by SHG-44 and SHG-44/ADM cells was $43.76 \pm 3.65$ and $45.87 \pm 3.36$ (relative fluorescence intensity), respectively. Following effusion, the residual amount of Rh123 in SGH-44 and SGH-44/ADM cells was $35.43 \pm 2.17$ and $18.49 \pm 3.54$, respectively. SHG44/ADM cells showed no difference in Rh123 ingestion as compared with SGH-44 cells, but significantly exuded more Rh123 than SGH-44 cells $(\mathrm{P}<0.01)$.

Analysis of apoptosis in SGH-44/ADM cells by flow cytometry and fluorescence microscopy. The apoptotic rate of SGH-44 cells increased from 3.52 to $70.97 \%$ when treated with ADM (Fig. 9). SGH-44/ADM cells were more resistant to ADM than SGH-44 cells, as the apoptotic rate showed a smaller increase from 3.19 to $15.89 \%$ when treated with ADM. However, the apoptotic rate of SGH-44/ADM cells was increased to $55.27 \%$ when treated with CsA, a MDR1 inhibitor. The apoptotic rate, determined by fluorescence microscopy, was accordant with that determined by flow cytometry (Fig. 10).

\section{Discussion}

In the present study, a stable MDR glioma cell line, SGH-44/ADM, was generated by impulse ADM treatment.
The effect of cryopreservation, recovery and withdrawal on SGH-44/ADM MDR cells was poor. SGH-44/ADM cells showed minimal differences as compared with parental cells, except that SGH-44/ADM cells were bigger in size, and with a wizened nucleus. However, SGH-44/ADM cells exhibited a slower growth rate and stronger excretion ability. As compared with SGH-44 cells, an increased number of SGH-44/ADM cells remained in $\mathrm{G} 1$ and $\mathrm{S}$ phase, as measured by flow cytometry. The MDR ability was associated with the upregulation of MDR1, PKC $\alpha$ and COX-2, but the expression of these genes was not associated with DNA methylation.

The cell model of MDR glioma has been described previously. Denecke et al (35) established a subline of the rat glioma cell line C6, named C6, 5x10(-7) Dox, by exposure to increasing doses of doxorubicin over five months. In addition to the typical cross-resistance to doxorubicin, daunorubicin, vincristine and etoposide, it was observed that a significant resistance of the C6, 5x10(-7) Dox cell line to irradiation was developed, which cannot be explained by Pgp expression. As well as the C6, 5x10(-7) Dox cell line, several other stable MDR human glioma cell lines had been previously established (36-38). All these previous studies failed to measure the resistance to ADM. Kang and Kang (38) established a dissoci- 
ated cell system of human glioblastoma multiforme (GBM) cells, A172. GBM2 cells were established with resistance to 1,3-bis(2-chloroethyl)-1-nitrosourea and expressed CD133, CD117, CD90, CD71 and CD45 cell-surface markers.

In the present study, Pgp was shown to be upregulated in the MDR cell line SGH-44/ADM, which was concordant to a previous study (35). COX-2 is an inducible isoform enzyme that functions to generate prostaglandins from arachidonic acid. It has been previously observed that enforced expression of COX-2 results in enhancement in MDR1 expression and functional activity, which suggests the existence of a causal link between COX-2 activity and MDR1 expression (39). The protein kinase C (PKC) family functions in regulating cell proliferation and death, and is a key protein in the signaling pathway linking epidermal growth factor receptor to mammalian target of rapamycin $(40,41)$. It had been observed that ribozymal inhibition of PKC $\alpha$ may trigger apoptosis in glioma cells (42). In the present study, PKC $\alpha$ and COX-2 were upregulated in SGH-44/ADM cells, which is consistent with previous studies $(39,42)$.

Methylation of $M D R 1, P K C \alpha$ and $C O X-2$ was not observed in the present study. The most significantly enriched pathway identified by MeDIP-Chip was SNARE interactions in vesicular transport $(\mathrm{P}=0.005)$. Three genes were involved in this pathway, including SNAP47, VAMP4 and VAMP3. The other significant pathways were sphingolipid metabolism, endocytosis, GPI-anchor biosynthesis, collecting duct acid secretion, pentose phosphate pathway and glycine, serine and threonine metabolism. Enriched genes associated with endocytosis were $C H M P 1 B, A R R B 2, P A R D 6 B, T G F B 1, V P S 4 B$ and $C B L B$.

The human VPS4B gene is a homolog of yeast VPS4, and shares a high degree of similarity with mouse suppressor of $\mathrm{K}^{+}$transport defect 1 , which is associated with transmembrane transport (43). VAMPs, NSF, SNAP and Annexins are the main components of the protein complex involved in the docking and fusion of synaptic vesicles with the presynaptic membrane (44), and may function in trans-Golgi network-to-endosome transport (45). CHMP1B is a peripherally-associated component of endosomal sorting. CHMP1B is required for transport complex III (ESCRT-III), which is involved in the formation and sorting of endosomal cargo proteins into multivesicular bodies (46). VPS4B, VAMPs and CHMPs are components of the ESCRT-III (46), which suggests that the major mechanism of MDR in glioma is exocytosis.

In conclusion, $\mathrm{SGH}-44 / \mathrm{ADM}$ cells were shown to have minimal differences from parental cells, except for a larger cell size and a wizened nucleus. A larger proportion of SGH-44/ADM cells remained in G1 and S phase, as compared with SGH-44 cells. The MDR ability of SGH-44/ADM cells was associated with the upregulation of MDR1, PKC $\alpha$ and COX-2. The expression of these genes was not associated with DNA methylation, which suggested that the mechanism of MDR in SGH-44/ADM is by active efflux rather than DNA methylation. In addition, certain components of immune-associated reactions, including positive regulation of cytotoxic T-cell differentiation, were significantly enriched. These findings may provide a novel insight into the MDR of human gliomas.

\section{Acknowledgements}

This study was supported by the Chongqing Natural Science Foundation of China (no. CSTC, 2009BB5262).

\section{References}

1. Mamelak AN and Jacoby DB: Targeted delivery of antitumoral therapy to glioma and other malignancies with synthetic chlorotoxin (TM-601). Expert Opin Drug Deliv 4: 175-186, 2007.

2. Weiss RB: The anthracyclines: will we ever find a better doxorubicin? Semin Oncol 19: 670-686, 1992.

3. Minotti G, Menna P, Salvatorelli E, Cairo G and Gianni L: Anthracyclines: molecular advances and pharmacologic developments in antitumor activity and cardiotoxicity. Pharmacol Rev 56: 185-229, 2004.

4. Peng X, Chen B, Lim CC and Sawyer DB: The cardiotoxicology of anthracycline chemotherapeutics: translating molecular mechanism into preventative medicine. Mol Interv 5: 163-171, 2005.

5. Stewart LA: Chemotherapy in adult high-grade glioma: a systematic review and meta-analysis of individual patient data from 12 randomised trials. Lancet 359: 1011-1018, 2002.

6. Lawson HC, Sampath P, Bohan E, et al: Interstitial chemotherapy for malignant gliomas: the Johns Hopkins experience. J Neurooncol 83: 61-70, 2007.

7. Johannessen T-CA, Bjerkvig $\mathrm{R}$ and Tysnes BB: DNA repair and cancer stem-like cells - potential partners in glioma drug resistance? Cancer Treat Rev 34: 558-567, 2008.

8. Bronger H, König J, Kopplow K, et al: ABCC drug efflux pumps and organic anion uptake transporters in human gliomas and the blood-tumor barrier. Cancer Res 65: 11419-11428, 2005.

9. Calatozzolo C, Gelati M, Ciusani E, et al: Expression of drug resistance proteins Pgp, MRP1, MRP3, MRP5 and GST-pi in human glioma. J Neurooncol 74: 113-121, 2005.

10. Dalton WS, Grogan TM, Meltzer PS, et al: Drug-resistance in multiple myeloma and non-Hodgkin's lymphoma: detection of P-glycoprotein and potential circumvention by addition of verapamil to chemotherapy. J Clin Oncol 7: 415-424, 1989.

11. Miller TP, Grogan TM, Dalton WS, et al: P-glycoprotein expression in malignant lymphoma and reversal of clinical drug resistance with chemotherapy plus high-dose verapamil. J Clin Oncol 9: 17-24, 1991.

12. Régina A, Demeule M, Laplante A, et al: Multidrug resistance in brain tumors: roles of the blood-brain barrier. Cancer Metastasis Rev 20: 13-25, 2001.

13. Mohri M, Nitta $\mathrm{H}$ and Yamashita J: Expression of multidrug resistance-associated protein (MRP) in human gliomas. J Neurooncol 49: 105-115, 2000.

14. Spiegl-Kreinecker S, Buchroithner J, Elbling L, et al: Expression and functional activity of the ABC-transporter proteins P-glycoprotein and multidrug-resistance protein 1 in human brain tumor cells and astrocytes. J Neurooncol 57: 27-36, 2002.

15. Herz J, Kowal RC, Goldstein JL and Brown MS: Proteolytic processing of the $600 \mathrm{kd}$ low density lipoprotein receptor-related protein (LRP) occurs in a trans-Golgi compartment. EMBO J 9: 1769-1776, 1990.

16. Pinzón-Daza M, Garzón R, Courard P, et al: The association of statins plus LDL receptor-targeted liposome-encapsulated doxorubicin increases in vitro drug delivery across blood-brain barrier cells. Br J Pharmacol 167:1431-1447, 2012.

17. Ratnasinghe D, Daschner PJ, Anver MR et al: Cyclooxygenase-2, P-glycoprotein-170 and drug resistance; is chemoprevention against multidrug resistance possible? Anticancer Res 21: 2141-2147, 2001.

18. Ali-Osman F, Caughlan J and Gray GS: Decreased DNA interstrand cross-linking and cytotoxicity induced in human brain tumor cells by 1,3-bis (2-chloroethyl)-1-nitrosourea after in vitro reaction with glutathione. Cancer Res 49: 5954-5958, 1989.

19. Chen C, Taniguchi T and D'Andrea A: The Fanconi anemia (FA) pathway confers glioma resistance to DNA alkylating agents. J Mol Med 85: 497-509, 2007.

20. Wu XG, Peng SB and Huang Q: Transcriptional regulation of breast cancer resistance protein. Yi Chuan 34: 1529-1536, 2012 (In Chinese).

21. Candelaria M, de la Cruz-Hernandez E, Taja-Chayeb L, et al: DNA methylation-independent reversion of gemcitabine resistance by hydralazine in cervical cancer cells. PLoS ONE 7: e29181, 2012. 
22. Ogawa T, Liggett TE, Melnikov AA, et al: Methylation of death-associated protein kinase is associated with cetuximab and erlotinib resistance. Cell Cycle 11: 1656-1663, 2012.

23. He J: Expression of glioma stem cell marker CD133 and O6-methylguanine-DNA methyltransferase is associated with resistance to radiotherapy in gliomas. Oncol Rep 26: 1305-1313,2011.

24. Wang B, Li Y, Tan Y, et al: Low-dose Cd induces hepatic gene hypermethylation, along with the persistent reduction of cell death and increase of cell proliferation in rats and mice. PLoS One 7: e33853, 2012.

25. Kanehisa M, Goto S, Kawashima S, Okuno Y and Hattori M: The KEGG resource for deciphering the genome. Nucleic Acids Res 32: D277-D280, 2004.

26. Yi M, Horton JD, Cohen JC, Hobbs HH and Stephens RM: WholePathwayScope: a comprehensive pathway-based analysis tool for high-throughput data. BMC Bioinformatics 7: 30, 2006.

27. Draghici S, Khatri P, Tarca AL, et al: A systems biology approach for pathway level analysis. Genome res 17: 1537-1545, 2007.

28. Gene Ontology Consortium: The Gene Ontology (GO) project in 2006. Nucleic Acids Res 34: D322-D326, 2006.

29. Ashburner M, Ball CA, Blake JA, et al: Gene ontology: tool for the unification of biology. The Gene Ontology Consortium. Nat Genet 25: 25-29, 2000.

30. Dupuy D, Bertin N, Hidalgo CA, et al: Genome-scale analysis of in vivo spatiotemporal promoter activity in Caenorhabditis elegans. Nat Biotechnol 25: 663-668, 2007.

31. Schlitt T, Palin K, Rung J, et al: From gene networks to gene function. Genome Res 13: 2568-2576, 2003.

32. Kim Y-J, Sah RLY, Doong J-YH and Grodzinsky AJ: Fluorometric assay of DNA in cartilage explants using Hoechst 33258. Anal Biochem 174: 168-176, 1988.

33. Zhang B, Kirov S, and Snoddy J: WebGestalt: an integrated system for exploring gene sets in various biological contexts. Nucleic Acids Res 33: W741-W7748, 2005.

34. Wang J, Duncan D, Shi Z and Zhang B: WEB-based GEne SeT AnaLysis Toolkit (WebGestalt): update 2013. Nucleic Acids Res 41: W77-W7783, 2013.

35. Denecke J, Fiedler K, Hacker-Klom U, et al: Multiple drug-resistant C6 glioma cells cross-resistant to irradiation. Anticancer Res 17: 4531-4534, 1997.
36. Shi L, Chen J, Yang J, et al: MiR-21 protected human glioblastoma U87MG cells from chemotherapeutic drug temozolomide induced apoptosis by decreasing $\mathrm{Bax} / \mathrm{Bcl}-2$ ratio and caspase- 3 activity. Brain Res 1352: 255-264, 2010.

37. Wang L, Xiong Y, Sun Y, et al: HLungDB: an integrated database of human lung cancer research. Nucleic Acids Res 38: D665-D669, 2010.

38. Kang MK and Kang SK: Tumorigenesis of chemotherapeutic drug-resistant cancer stem-like cells in brain glioma. Stem Cells Dev 16: 837-847, 2007.

39. Sorokin A: Cyclooxygenase-2: potential role in regulation of drug efflux and multidrug resistance phenotype. Current Pharmaceutical Design 10: 647-657, 2004.

40. Fan Q-W, Cheng C, Knight ZA, et al: EGFR signals to mTOR through PKC and independently of Akt in glioma. Sci Signal 2: ra4, 2009.

41. Basu A: PKC and resistance to chemotherapeutic agents. In: Protein Kinase $\mathrm{C}$ in Cancer Signaling and Therapy. Kazanietz MG (ed.) Humana Press, pp 409-429, 2010.

42. Leirdal $M$ and Sioud M: Ribozyme inhibition of the protein kinase $\mathrm{C}$ alpha triggers apoptosis in glioma cells. Br J Cancer 80: 1558-1564, 1999.

43. Fujita H, Yamanaka M, Imamura K, et al: A dominant negative form of the AAA ATPase SKD1/VPS4 impairs membrane trafficking out of endosomal/lysosomal compartments: class E vps phenotype in mammalian cells. J Cell Sci 116: 401-414, 2003.

44. Schnitzer JE, Liu J and Oh P: Endothelial caveolae have the molecular transport machinery for vesicle budding, docking, and fusion including VAMP, NSF, SNAP, annexins, and GTPases. J Biol Chem 270: 14399-14404, 1995.

45. Kawano M, Kumagai K, Nishijima M and Hanada K: Efficient trafficking of ceramide from the endoplasmic reticulum to the Golgi apparatus requires a VAMP-associated protein-interacting FFAT motif of CERT. J Biol Chem 281: 30279-30288, 2006.

46. Guizetti J and Gerlich DW: ESCRT-III polymers in membrane neck constriction. Trends Cell Biol 22: 133-140, 2012. 